\title{
Association of Accelerated Long-term Forgetting and Senescence-related Blood-borne Factors in Asymptomatic Individuals From Families With Autosomal Dominant Alzheimer's Disease
}

\author{
Jianwei Yang \\ Xuanwu Hospital \\ Chaojun Kong \\ Xuanwu Hospital \\ Longfei Jia \\ Xuanwu Hospital \\ Tingting Li \\ Xuanwu Hospital \\ Meina Quan \\ Xuanwu Hospital \\ Yan Li \\ Xuanwu Hospital \\ Diyang Lyu \\ Xuanwu Hospital \\ Fangyu Li \\ Xuanwu Hospital \\ Hongmei Jin \\ Xuanwu Hospital \\ Ying Li \\ Xuanwu Hospital \\ Qigeng Wang \\ Xuanwu Hospital \\ Jianping Jia ( $\nabla$ jiajp@vip.126.com ) \\ Xuanwu Hospital https://orcid.org/0000-0003-4624-0336
}

\section{Research Article}

Keywords: Alzheimer's disease, accelerated long-term forgetting, blood-borne factors, senescence, biomarkers 
Posted Date: March 10th, 2021

DOI: https://doi.org/10.21203/rs.3.rs-282897/v1

License: (c) (i) This work is licensed under a Creative Commons Attribution 4.0 International License. Read Full License 


\section{Abstract}

Background: Accelerated long-term forgetting has been identified in preclinical Alzheimer's disease (AD), and is attributed to a selective impairment of memory consolidation in which hippocampus plays a key role. As blood may contain multiple senescence-related factors that involved in neurogenesis and synaptic plasticity in the hippocampus, we tested whether there is an association between blood-borne factors and accelerated long-term forgetting in asymptomatic individuals from families with autosomal dominant AD (ADAD).

Methods: We analyzed data of 39 asymptomatic participants ( $\mathrm{n}=18$ ADAD mutation carriers, $\mathrm{n}=21$ noncarriers) from the Chinese Familial Alzheimer's Disease Network (CFAN) study. Long-term forgetting rates were calculated based on recall or recognition of two materials (word list and complex figure) at three delays comprising immediate, $30 \mathrm{~min}$ and 7 days. Peripheral blood concentrations of candidate pro-aging factors (C-C motif ligand 11 [CCL11] and monocyte chemotactic protein 1 [MCP1]) and rejuvenation factors (growth differentiation factor 11 [GDF11], thrombospondin-4 [THBS4], and secreted protein acidic and rich in cysteine like 1 [SPARCL1]) were evaluated in all participants.

Results: Despite normal performance on standard 30-min delayed testing, mutation carriers exhibited accelerated forgetting of verbal and visual material over 7 days in comparison with matched non-carriers. In the whole sample, lower plasma THBS4 was associated with accelerated long-term forgetting in list recall $(\beta=-0.46, p=0.002)$, figure recall $(\beta=-0.44, p=0.004)$ and list recognition $(\beta=-0.37, p=0.010)$. Additionally, higher plasma GDF11 and CCL11 were both associated with accelerated long-term forgetting (GDF11 versus figure recall: $\beta=0.39, p=0.007$; CCL11 versus list recognition: $\beta=0.44, p=0.002$ ).

Conclusions: Accelerated long-term forgetting is a cognitive feature of presymptomatic AD. Senescencerelated blood-borne factors, especially THBS4, GDF11, and CCL11, may be promising biomarkers for the prediction of accelerated long-term forgetting.

\section{Background}

Alzheimer's disease (AD) is a clear and present concern to public health in China and internationally, and has a profound socio-economic impact ${ }^{[1]}$. Early identification of $A D$ has become a priority in research, as it may open the door to preventive approaches ${ }^{[2]}$. The fact that $A D$ pathological changes develop decades before visible cognitive impairments might reflect that the sensitivities of the cognitive assays currently used are not sufficient enough ${ }^{[3,4]}$. In this context, increasing evidence has suggested that accelerated long-term forgetting would be a subtle cognitive impairment in presymptomatic autosomal dominant AD (ADAD) mutation carriers ${ }^{[5,6]}$. This memory impairment was thought to be attributed to a failure of memory consolidation process, which was primarily governed by the medial temporal lobe (MTL), especially the hippocampus ${ }^{[7]}$. 
A flurry of studies has demonstrated that specific factors are present in the blood that directly affect neurogenesis and synaptic plasticity in the hippocampus, and thus regulate memory and cognitive function ${ }^{[8-12]}$. For example, initial studies in mouse models have identified pro-aging factors (monocyte chemotactic protein 1 [MCP1], and C-C motif ligand 11 [CCL11]) ${ }^{[8]}$ and rejuvenating factor (growth differentiation factor 11 [GDF11] ${ }^{[9]}$ that correlated with hippocampal neurogenesis and cognitive function. More recently, both thrombospondin-4 (THBS4) and secreted protein acidic and rich in cysteine like 1 (SPARCL1) were found to represent rejuvenation factors that enhance synaptic connectivity ${ }^{[10]}$. These results provide support for the idea that senescence-related blood-borne factors would be served as sensitive predictors of cognitive function (specifically hippocampal memory). However, the relationship of blood-borne factors and cognitive deficits in patients with AD has not been extensively studied so far, and few studies have examined these factors with respect to subtle cognitive change, especially hippocampal-dependent memory impairments, in the preclinical stage.

The goal of this study was to explore whether blood-borne factors were associated with long-term forgetting rates in asymptomatic carriers and non-carriers of ADAD mutations. We expected to find that higher pro-aging factors or lower rejuvenation factors would be associated with accelerated long-term forgetting in the early stage of $A D$.

\section{Methods}

\section{Participants}

All participants in this study were recruited from the Chinese Familial Alzheimer's Disease Network (CFAN), which is a multicenter, longitudinal cohort used to collect the hereditary and clinical profile of familial $A D^{[13]}$. We included 18 mutation carriers with a known causative mutation of $A D$ in the amyloid precursor protein (APP), presenilin 1(PSEN1), or presenilin 2 (PSEN2) genes and 21 non-carrier family members. Participants were matched across basic aspects of demography, i.e., age, sex and education. The estimated proximity to onset for mutation carriers of ADAD was calculated as the age of the participant at assessment minus the age of the parent at symptom onset ${ }^{[14]}$. All participants were blind to their genetic status, in accordance with cultural norms and ethical regulations in this community. Inclusion criteria were: Clinical Dementia Rating (CDR) global score $=0$ and Mini-Mental State Examination (MMSE) score $\geq 26$. None of the participants reported complaints of anxiety or depressive symptoms. This study was approved by the Medical Research Ethics Committee of Xuanwu Hospital, Capital Medical University. All participants in the study provided written informed consent.

\section{Standard neuropsychological testing}

Clinical disease severity was rated using the CDR scale, which incorporates information from both participant and informant on day-to-day cognition ${ }^{[15]}$. The MMSE ${ }^{[16]}$ and Montreal Cognitive Assessment $(\mathrm{MoCA})^{[17]}$ were used to evaluate global cognitive function. Self-report of subject cognitive concerns was measured with the participant (MyCog) version of Subjective Cognitive Decline Questionnaire (SCD-Q), 
which quantifies perceived subjective cognitive decline over the last 2 years ${ }^{[18]}$. Subscores for memory (items 1-11), language (items 12-17), and executive (items 18-24) functions were also computed.

\section{Assessment of long-term forgetting rates}

The WHO-University of California Los Angeles-Auditory Verbal Learning test (WHO-UCLA-AVLT) was used to assess verbal learning, recall and recognition performances ${ }^{[19]}$. This version of auditory verbal learning test consists of a list of 15 non-associated words that is presented orally. During this test, participants had to learn the material to a minimum required accuracy of $80 \%$ at free recall over a minimum of 3 and a maximum of 10 trials. The Rey-Osterrieth Complex Figure (ROCF) test was used to examine visuospatial copy and recall[20]. During this test, participants were asked to copy the figure as accurately as possible, and were instructed that they would later be asked to draw it from memory. Both the figure and copy were then removed from view and participants were asked to draw the figure again from memory.

At 30 min and 7 days later, participants were once again asked to recall the word list and to draw the figure from memory in as much detail as they could remember. For the word list, a recognition test was also performed during which the 15 target words were presented among 15 distracters. There was no recognition for visual memory test. Participants were not informed about the 7-day follow-up assessment, which was conducted over the phone. This ensures that participants would not be motivated to rehearse. The 7-day follow-up figure assessments were returned by mail. The long-term forgetting rate is defined as the percentage of information lost between 30-min and 7-day delays, as follows: (1-performance at 7-day delay/ performance at 30-min delay) $\times 100^{[21]}$.

\section{Blood collection and analysis}

Non-fasting venous blood for plasma analyses was collected in EDTA-containing tubes, which were then centrifuged at $2000 \times \mathrm{g}$ for 15 minutes at $4^{\circ} \mathrm{C}$. For serum analyses, blood samples were collected in tubes without anticoagulant and centrifuged after removal of clots of blood at room temperature for a half hour. EDTA plasma and serum samples were stored in single-use aliquots at $-80^{\circ} \mathrm{C}$ until biochemical analysis.

Plasma CCL11, MCP1, GDF11, THBS4, and serum SPARCL1 concentrations were detected using enzymelinked immunosorbent assay (ELISA, Supplementary Table 1). All analyses were performed in duplicate, according to manufacturer's published protocols. Final data were examined for extreme outliers, and samples with $>50 \times$ the upper interquartile range were also excluded ( $n=1$ on MCP1).

\section{Statistical analyses}

Group differences in participant characteristics were assessed using independent t-tests (or MannWhitney $U$ tests) for continuous and $\chi^{2}$-test for categorical variables. Differences in long-term memory performance were compared between groups using multivariate linear regression, adjusted for age, sex 
and education. Receiver operating characteristic (ROC) curve analysis was used to determine the ability of long-term forgetting rates to discriminate between mutation carriers and non-carriers. Spearman's correlations were used to test associations among long-term forgetting rates, blood-borne factors, estimated proximity to onset and subject cognitive concerns in mutation carriers. To assess factors that could predict long-term forgetting rates, multivariate linear regression was performed for the whole sample with age, sex, education and blood-borne factors included in the model, using a stepwise method. The statistical analyses were performed with IBM SPSS software. All tests were two-sided, and $p$ values less than 0.05 were considered statistically significant.

\section{Results}

\section{Sample characteristics}

Demographic, clinical, and neuropsychological characteristics of sample are presented in Table 1. As anticipated, mutation carriers did not differ from non-carriers in basic demographic variables, such as age, sex, and education attainment. There were no significant differences regarding the global level of cognition between carriers and non-carriers. For the subjective cognitive ratings, mutation carriers showed a trend towards higher total MyCog scores compared to non-carriers $(p=0.068)$, while the cognitive subdomains of MyCog did not differ between the two groups. There was no difference in plasma concentrations of CCL11, MCP1, GDF11 and THBS4 between mutation carriers and non-carriers. The between-group difference of serum SPARCL1 concentration was not significant, either. 
Table 1

Demographic, clinical and neuropsychological characteristics of study participants

Variable Mutation carriers $(n=18) \quad$ Non-carriers $(n=21) \quad$ Difference $(p)$

Demographic data

\begin{tabular}{llll} 
Female & $8(44.4)$ & $11(52.4)$ & 0.751 \\
\hline Age, y & $38.0(35.8-43.0)$ & $35.0(33.0-48.0)$ & 0.282 \\
\hline Estimated proximity to onset, y & $11.6(5.3)$ & NA & \\
\hline Education, y & $14.0(11.0-15.3)$ & $16.0(11.5-16.5)$ & 0.094
\end{tabular}

\section{Cognitive level (global)}

$\begin{array}{llll}\text { MMSE score } & 29.5(29.0-30.0) & 30.0(29.0-30.0) & 0.512 \\ \text { MoCA score } & 28.0(27.0-28.0) & 27.0(27.0-28.5) & 0.606\end{array}$

\section{SCD-Q MyCog}

\begin{tabular}{llll} 
Total score & $6.0(2.0-9.3)$ & $2.0(0.5-7.0)$ & 0.068 \\
\hline Memory subscore & $4.0(1.8-6.0)$ & $2.0(0-4.5)$ & 0.139 \\
\hline Language subscore & $1.0(0-2.0)$ & $0(0-1.0)$ & 0.131 \\
\hline Executive subscore & $1.0(0-1.5)$ & $0(0-1.0)$ & 0.150
\end{tabular}

\section{Senescence-related factor}

\begin{tabular}{llll} 
CCL11, pg/ml & $83.85(30.00)$ & $72.39(23.15)$ & 0.187 \\
\hline MCP1, pg/ml & $173.96(54.56)$ & $162.24(63.53)$ & 0.551 \\
\hline GDF11, pg/ml & $89.08(47.06)$ & $73.08(34.76)$ & 0.231 \\
\hline THBS4, ng/ml & $128.34(49.20)$ & $168.08(81.27)$ & 0.079 \\
\hline SPARCL $1, \mathrm{ng} / \mathrm{ml}$ & $5.59(1.94)$ & $5.32(1.86)$ & 0.666
\end{tabular}

Data are $\mathrm{n}(\%)$, median (IQR) or mean (SD).

Abbreviations: CCL 11 C-C motif ligand 11, GDF11 growth differentiation factor 11, MCP1 monocyte chemotactic protein 1, MMSE Mini-Mental State Examination, MoCA Montreal Cognitive Assessment, $N A$ not applicable, $S C D-Q$ Subjective Cognitive Decline Questionnaire, SPARCL 1 secreted protein acidic and rich in cysteine like 1, THBS4 thrombospondin-4

\section{Differences between groups in long-term memory performance}

Age-, sex- and education-adjusted comparisons of long-term memory performance revealed significantly poorer retention of material at 7-day recall scores for list and figure in the mutation carriers compared to 
non-carriers (Table 2). Mutation carriers also did worse than non-carriers on 7-day list recognition. No significant group differences were found for the initial score and the number of learning trials. In addition, recall and recognition score at $30 \mathrm{~min}$ for list, as well as recall score at $30 \mathrm{~min}$ for figure did not differ between the two groups. 
Table 2

Group differences in long-term memory performance

$\begin{array}{llll}\begin{array}{l}\text { Mutation } \\ \text { carriers }\end{array} & \text { Non-carriers } & \begin{array}{l}p \\ \text { value }\end{array} & \begin{array}{l}\text { Difference in } \\ \text { mean }\end{array} \\ & & 95 \% \mathrm{Cl}\end{array}$

\section{Learning}

List learning trials

$4.0(4.0-5.0)$

$4.0(4.0-5.0)$

$0.981-0.01$

-0.71 to

0.69

List learning score

$80.0(80.0-$

86.7)

$86.7(80.0-$

86.7)

-4.55 to

1.78

Figure learning score

$72.9(63.2-$

$69.4(60.4-$

76.4)

-2.61 to

80.6)

0.193

4.95

12.51

\section{0-min retention}

List 30-min recall

73.3 (66.7-

73.3 (70.0-

0.227

$-3.46$

-9.15 to

80.0)

80.0)

2.25

Figure $30-\mathrm{min}$ recall

69.4 (56.9-

79.5)

66.7 (56.9-

72.2)

0.418

3.36

-4.96 to

90.0 (73.3-

93.3 (83.3-

93.3)

0.294

$-3.64$

11.67

List 30-min recognition

93.3)

-10.57 to

3.29

\section{7-day retention}

List 7-day recall

$53.3(40.0-$ 60.0)

$66.7(60.0-$ 80.0)

-22.23 to

$-8.46$

Figure 7-day recall

38.9 (33.3-

$58.3(47.9-$

60.4)

-19.20 to

52.8)

$80.0(73.3-$

$70.0(53.3-$

$90.0)$

$0.006 \quad-11.52$

$-5.42$

List 7-day recognition

$80.0)$

-19.50 to

$-3.53$

\section{Long-term forgetting rate}

Forgetting rate in list recall

Forgetting rate in figure recall

Forgetting rate in list recognition
$26.1(18.2-$ 34.1)

$36.3(28.4-$ 48.1)

20.7 (14.328.9)
9.1 (0.0-

14.1)

10.5 (7.1-

20.4)

7.7 (0.0-

18.3)
$<0.001 \quad 17.26$

$<0.001 \quad 22.30$

$0.003 \quad 10.07$

Data are uncorrected group medians (IQR). Differences in means, $95 \% \mathrm{Cls}$, and $p$ values are adjusted for age, sex and education. 
ROC analysis for discrimination between mutation carriers and non-carriers for long-term forgetting rates revealed an AUC of $0.881(95 \% \mathrm{Cl} 0.774-0.988)$ for list recall, $0.894(0.783-1.000)$ for figure recall, and 0.790 (0.645-0.934) for list recognition (Fig. 1).

\section{Association of blood-borne factors with long-term forgetting rates}

There was a significant association between blood-borne factors and long-term forgetting rates in the whole sample, adjusted for age, sex, and education (Table 3). Lower plasma THBS4 was associated with accelerated long-term forgetting in list recall $(\beta=-0.46, p=0.002)$, figure recall $(\beta=-0.44, p=0.004)$, and list recognition $(\beta=-0.37, p=0.010)$. In addition, there were significant associations of higher plasma GDF11 with accelerated forgetting in figure recall $(\beta=0.39, p=0.007)$, and higher plasma CCL11 with accelerated forgetting in list recognition $(\beta=0.44, p=0.002)$. 
Table 3

Association of blood-borne factors with long-term forgetting rates

\begin{tabular}{llllllll}
\hline Dependent variables & Independent factors & B & B SE & $\boldsymbol{\beta}$ & $\boldsymbol{p}$ value & $\mathbf{R}^{2}$ \\
\hline The whole sample & & & & & & \\
\hline Forgetting rate in list recall & age & 1.14 & 0.22 & 0.69 & $<0.001$ & 0.43 \\
\hline & THBS4 & -0.10 & 0.03 & -0.46 & 0.002 & \\
\hline Forgetting rate in figure recall & GDF11 & -10.53 & 3.93 & -0.36 & 0.011 & \\
\hline & THBS4 & 0.15 & 0.05 & 0.39 & 0.007 & 0.34 \\
\hline Forgetting rate in list recognition & CCL11 & -0.10 & 0.03 & -0.44 & 0.004 & \\
\hline & THBS4 & -8.94 & 4.35 & -0.29 & 0.048 & \\
\hline Mutation carriers & age & 0.19 & 0.06 & 0.44 & 0.002 & 0.35 \\
\hline Forgetting rate in list recall & age & -0.06 & 0.02 & -0.37 & 0.010 & \\
\hline Forgetting rate in figure recall & GDF11 & 0.42 & 0.18 & 0.32 & 0.025 & \\
\hline Non-carriers & & & & & & \\
\hline Forgetting rate in list recall & MCP-1 & 1.16 & 0.33 & 0.67 & 0.003 & 0.41 \\
\hline Forgetting rate in figure recall & THBS4 & 0.17 & 0.06 & 0.59 & 0.012 & 0.31 \\
\hline Forgetting rate in list recognition & CCL11 & & & & & \\
\hline Sex & & -0.05 & 0.02 & -0.44 & 0.046 & 0.15 \\
\hline
\end{tabular}

Results of multivariate regression analysis (stepwise) with the blood-borne factors as independent factors, and the long-term retrieval rates as dependent variables. Only statistically significant associations are listed $(p<0.05)$.

Abbreviations: CCL 11 C-C motif ligand 11, GDF11 growth differentiation factor 11, MCP1 monocyte chemotactic protein 1, THBS4 thrombospondin-4

In mutation carriers, there was a consistent association between higher plasma GDF11 and accelerated forgetting in figure recall $(\beta=0.59, p=0.012)$, but no evidence of correlations between any other bloodborne factors and long-term forgetting rates.

Associations between estimated proximity to onset and long-term forgetting rates or blood-borne factors 
In mutation carriers, there was an association between estimated proximity to onset and long-term forgetting rates for list recall $(R=-0.69, p=0.002$; Fig. $2 A)$ and recognition $(R=-0.78, p<0.001$; Fig. 2B), such that those who were close to clinical onset showed faster forgetting rates. However, no significant relationships were found for the figure recall $(R=-0.44, p=0.071)$.

As for blood-borne factors, there was an association between estimated proximity to onset and plasma THBS4 $(\mathrm{R}=0.51, p=0.032)$, with the lower of plasma THBS4 concentrations increasing with proximity to symptom onset (Fig. 2C). None of the other blood-borne factors showed a significant association with estimated proximity to onset.

\section{Association between long-term forgetting rates and subject cognitive concerns}

In the whole sample and mutation carriers, an association existed between higher total score or memory subscore of SCD-Q MyCog and accelerated long-term forgetting, which reached statistical significance for list recall, but not for figure recall and list recognition (Supplementary Table 2).

\section{Discussion}

The present study added more evidence to existing literature characterizing accelerated long-term forgetting as a subclinical cognitive impairment in ADAD mutation carriers. Further, we showed that there is an association between senescence-related blood-borne factors and accelerated long-term forgetting in pooled participants with mutation carriers and non-carriers. To our knowledge, this study is the first that attempt to determine the association of blood-borne factors and accelerated long-term forgetting in asymptomatic individuals. Our findings revealed the promise of blood-borne factors as potential biomarkers for the prediction of accelerated long-term forgetting.

In this study, we have demonstrated that accelerated long-term forgetting is an early feature of presymptomatic ADAD mutation carriers, who were on average 12 years before their predicted age at symptom onset. Our finding was consistent with those reported in previous studies that accelerated longterm forgetting is present in presymptomatic ADAD mutation carriers, and might provide a means of discriminating presymptomatic $A D$ from controls ${ }^{[5,6]}$. In addition, accelerated long-term forgetting was associated with greater subjective cognitive concerns in mutation carriers. This association is also consistent with findings from the previous study ${ }^{[5]}$. As for sporadic $A D$, accelerated long-term forgetting was also found in asymptomatic $A P O E \& 4$ carriers with a mean age well below their predicted age at symptom onset ${ }^{[22,23]}$. More recently, a longitudinal study showed that accelerated long-term forgetting over 4 weeks could predict cognitive decline in healthy older people ${ }^{[7]}$. Together, these results highlight the potential of accelerated long-term forgetting in screening for patient candidates and guiding the selection of outcome measures in future preclinical trials.

The molecular mechanisms underlying accelerated long-term forgetting are not fully understood. The pattern of relatively intact retention over short delays, coupled with poor retention after longer delays is 
suggestive of a selective impairment of memory consolidation ${ }^{[24]}$. Memory consolidation is the process that transform the labile memory traces into stable long-term memories, with the hippocampus and MTL cortices known to play an important part ${ }^{[25]}$. Therefore, it is currently believed that impaired memory consolidation caused by Hippocampal dysfunction might produce accelerated long-term forgetting. For example, a recent study has shown that both seizures and interictal spikes in hippocampus during sleep impair long-term memory consolidation, supporting the notion that hippocampal dysfunction causes accelerated long-term forgetting ${ }^{[26]}$.

Hippocampal dysfunction has been used to explain the phenomenon of accelerated long-term forgetting, as mentioned above. Thus, blood-borne factors that contribute to neurogenesis and synaptic plasticity in the hippocampus could provide useful biomarkers for accelerated long-term forgetting. In this study, lower plasma THBS4 was significantly associated with accelerated long-term forgetting.

Thrombospondins, including THBS 1-5, are large secreted extracellular matrix proteins that have been previously identified as possible synaptogenic factors in the central nervous system ${ }^{[27,28]}$. In a recent in vitro study, THBS4 was found to directly stimulate dendritogenesis and synapse formation in cultured neurons ${ }^{[10]}$. Given the fact that it may be possible for THBS4 to be transported from blood into the brain parenchyma and directly activate synapse formation and function ${ }^{[10]}$, lower levels of plasma THBS4 in relation to accelerated long-term forgetting are to be expected.

Additionally, we found that higher plasma GDF11 is associated with accelerated long-term forgetting. This is an unexpected finding since GDF11 may enhance neurogenesis in the adult brain ${ }^{[9]}$ and reduced $A D$ pathology has been observed in association with increasing plasma GDF11 concentrations in AD animal models ${ }^{[29]}$. However, the concentration of GDF11 in plasma was determined using antibody-based method in our study, which may be thwarted by insufficient specificity due to the high homology between GDF11 and MSTN/GDF8 ${ }^{[30]}$. Furthermore, a previous antibody-reliant study did not detect correlations between plasma GDF11 and cognitive impairment, probably for the same reason ${ }^{[31]}$. Thus, further studies are required to determine whether and how GDF11 in blood may influence cognitive function and memory performance.

In the present study, higher plasma CCL11 has also been found to be correlated with accelerated longterm forgetting. This finding is consistent with prior studies using animal models of aging demonstrating increased plasma CCL11 correlate with reduced neurogenesis and impaired learning and memory ${ }^{[8]}$. Previous studies have also suggested that serum concentration of CCL11 is significantly higher in AD patients than in normal controls ${ }^{[32]}$, and CCL 11 is negatively associated with cognitive performance in rural dwellers ${ }^{[33]}$. It is worth mentioning that circulating CCL11 can access many regions of the brain and exert negative influences in the central nervous system via transport across the blood-brain barrier ${ }^{[34]}$. Thus, blood-borne CCL11 may be involved in the process of AD in aging, and peripheral CCL11 concentrations might be able to predict cognitive impairment.

\section{Limitations}


This study is not without limitations. The first is the relatively small sample size in the present study, which may affect the quality of the statistical analysis. As such, validation in future studies with larger sample sizes is needed to confirm our findings. Another important limitation is the absence of longitudinal assessments of the participants, and we failed to infer causality with the presented data. To fully appreciate the dynamic changes of blood-borne factors and accelerated long-term forgetting along the disease continuum, further follow-up of these participants is needed. Finally, some factors cannot be readily controlled, such as practice effect, 7-day testing environment, and participant's sleep quality. These methodological issues are important to take into account when investigating accelerated long-term forgetting in our future studies.

\section{Conclusions}

In summary, our study suggests that accelerated long-term forgetting, as a hippocampal-dependent memory impairment, is present at earlier stage in the AD continuum. Senescence-related blood-borne factors that correlated with hippocampal dysfunction, including THBS4, GDF11, and CCL11, may present ideal biomarkers for accelerated long-term forgetting. Our findings underscore the idea that blood-borne factors may provide insights into subtle cognitive decline in presymptomatic $A D$, which deserves further studies in the next.

\section{Abbreviations}

AD: Alzheimer's disease; ADAD: autosomal dominant autosomal dominant; APP: amyloid precursor protein; CCL11: C-C motif ligand 11; CDR: Clinical Dementia Rating; CFAN: Chinese Familial Alzheimer's Disease Network; ELISA: enzyme-linked immunosorbent assay; GDF11: growth differentiation factor 11; MCP1: monocyte chemotactic protein 1; MMSE: Mini-Mental State Examination; MoCA: Montreal Cognitive Assessment; PSEN1: presenilin 1; PSEN2: presenilin 2; ROCF: Rey-Osterrieth Complex Figure; ROC: receiver operating characteristic; SCD-Q: Subjective Cognitive Decline Questionnaire; SPARCL1: secreted protein acidic and rich in cysteine like 1; THBS4: thrombospondin-4; WHO-UCLA-AVLT: WHOUniversity of California Los Angeles-Auditory Verbal Learning test

\section{Declarations}

\section{Acknowledgements}

We would like to thank all the researchers in the CFAN study (http://www.chinacfan.org). We thank the participants and their families for their generous support of this study, and our clinical colleagues across China for referring patients.

\section{Authors' contributions}

YJ and $\mathrm{JJ}$ designed the research and wrote the manuscript. YJ, CK, LJ, TL, HJ, YL and QW contributed to participant recruitment and data collection. YJ, CK, LJ, MQ, YL, DL, FL and JJ analyzed and interpreted 
the data. YJ, CK, LJ, MQ, YL, DL and JJ reviewed and revised the manuscript. All authors read and approved the final manuscript.

\section{Funding}

This study was supported by the Key Project of the National Natural Science Foundation of China (81530036); the National Key Scientific Instrument and Equipment Development Project (31627803); the Key Project of the National Natural Science Foundation of China (U20A20354); Beijing Scholars Program; Beijing Brain Initiative from Beijing Municipal Science \& Technology Commission (Z201100005520016, Z201100005520017).

\section{Availability of data and materials}

The data generated and analyzed in the current study can be made available upon request to the corresponding author and the CFAN committee in order to ensure that the privacy of the CFAN participants is protected.

\section{Ethics approval and consent to participate}

The study procedures were approved by the Medical Research Ethics Committee at Xuanwu Hospital, and written informed consents were obtained from all participants.

\section{Consent for publication}

Not applicable.

\section{Competing interests}

The authors declare that they have no competing interests.

\section{References}

1. Jia L, Quan M, Fu Y, Zhao T, Li Y, Wei C, et al. Dementia in China: epidemiology, clinical management, and research advances. Lancet Neurol. 2020;19(1):81-92.

2. Xiong C, Luo J, Agboola F, Li Y, Albert M, Johnson SC, et al. A harmonized longitudinal biomarkers and cognition database for assessing the natural history of preclinical Alzheimer's disease from young adulthood and for designing prevention trials. Alzheimers Dement. 2019;15(11):1448-57.

3. Bateman RJ, Xiong C, Benzinger TL, Fagan AM, Goate A, Fox NC, et al. Clinical and biomarker changes in dominantly inherited Alzheimer's disease. N Engl J Med. 2012;367(9):795-804.

4. Caselli RJ, Langlais BT, Dueck AC, Chen Y, Su Y, Locke DEC, et al. Neuropsychological decline up to 20 years before incident mild cognitive impairment. Alzheimers Dement. 2020;16(3):512-23.

5. Weston PSJ, Nicholas JM, Henley SMD, Liang Y, Macpherson K, Donnachie E, et al. Accelerated longterm forgetting in presymptomatic autosomal dominant Alzheimer's disease: a cross-sectional study. 
Lancet Neurol. 2018;17(2):123-32.

6. O'Connor A, Weston PSJ, Pavisic IM, Ryan NS, Collins JD, Lu K, et al. Quantitative detection and staging of presymptomatic cognitive decline in familial Alzheimer's disease: a retrospective cohort analysis. Alzheimers Res Ther. 2020;12(1):126.

7. Wearn AR, Saunders-Jennings E, Nurdal V, Hadley E, Knight MJ, Newson M, et al. Accelerated longterm forgetting in healthy older adults predicts cognitive decline over 1 year. Alzheimers Res Ther. 2020;12(1):119.

8. Villeda SA, Luo J, Mosher KI, Zou B, Britschgi M, Bieri G, et al. The ageing systemic milieu negatively regulates neurogenesis and cognitive function. Nature. 2011;477(7362):90-4.

9. Katsimpardi L, Litterman NK, Schein PA, Miller CM, Loffredo FS, Wojtkiewicz GR, et al. Vascular and neurogenic rejuvenation of the aging mouse brain by young systemic factors. Science. 2014;344(6184):630-4.

10. Gan KJ, Sudhof TC. Specific factors in blood from young but not old mice directly promote synapse formation and NMDA-receptor recruitment. Proc Natl Acad Sci U S A. 2019;116(25):12524-33.

11. Castellano JM. Blood-Based Therapies to Combat Aging. Gerontology. 2019;65(1):84-9.

12. Imbimbo BP, Ippati S, Ceravolo F, Watling M. Perspective: Is therapeutic plasma exchange a viable option for treating Alzheimer's disease? Alzheimers Dement (N Y). 2020;6(1):e12004.

13. Jia L, Fu Y, Shen L, Zhang H, Zhu M, Qiu Q, et al. PSEN1, PSEN2, and APP mutations in 404 Chinese pedigrees with familial Alzheimer's disease. Alzheimers Dement. 2020;16(1):178-91.

14. Preische O, Schultz SA, Apel A, Kuhle J, Kaeser SA, Barro C, et al. Serum neurofilament dynamics predicts neurodegeneration and clinical progression in presymptomatic Alzheimer's disease. Nat Med. 2019;25(2):277-83.

15. Morris JC. The Clinical Dementia Rating (CDR): current version and scoring rules. Neurology. 1993;43(11):2412-4.

16. Folstein MF, Folstein SE, McHugh PR. "Mini-mental state". A practical method for grading the cognitive state of patients for the clinician. J Psychiatr Res. 1975;12(3):189-98.

17. Nasreddine ZS, Phillips NA, Bedirian V, Charbonneau S, Whitehead V, Collin I, et al. The Montreal Cognitive Assessment, MoCA: a brief screening tool for mild cognitive impairment. J Am Geriatr Soc. 2005;53(4):695-9.

18. Rami L, Mollica MA, Garcia-Sanchez C, Saldana J, Sanchez B, Sala I, et al. The Subjective Cognitive Decline Questionnaire (SCD-Q): a validation study. J Alzheimers Dis. 2014;41(2):453-66.

19. Maj M, Satz P, Janssen R, Zaudig M, Starace F, D'Elia L, et al. WHO Neuropsychiatric AIDS study, cross-sectional phase II. Neuropsychological and neurological findings. Arch Gen Psychiatry. 1994;51(1):51-61.

20. Buhlmann U, Deckersbach T, Engelhard I, Cook LM, Rauch SL, Kathmann N, et al. Cognitive retraining for organizational impairment in obsessive-compulsive disorder. Psychiatry Res. 2006;144(2-3):10916. 
21. Geurts S, van der Werf SP, Kwa VIH, Kessels RPC. Accelerated long-term forgetting after TIA or minor stroke: A more sensitive measure for detecting subtle memory dysfunction? Cortex. 2019;110:150-6.

22. Zimmermann JF, Butler CR. Accelerated long-term forgetting in asymptomatic APOE epsilon4 carriers. Lancet Neurol. 2018;17(5):394-5.

23. Tort-Merino A, Laine $M$, Valech $N$, Olives J, Leon M, Ecay-Torres $M$, et al. Accelerated long-term forgetting over three months in asymptomatic APOE varepsilon4 carriers. Ann Clin Transl Neurol. 2020.

24. Hoefeijzers S, Dewar M, Della Sala S, Zeman A, Butler C. Accelerated long-term forgetting in transient epileptic amnesia: an acquisition or consolidation deficit? Neuropsychologia. 2013;51(8):1549-55.

25. Squire LR, Genzel L, Wixted JT, Morris RG. Memory consolidation. Cold Spring Harb Perspect Biol. 2015;7(8):a021766.

26. Lambert I, Tramoni-Negre E, Lagarde S, Roehri N, Giusiano B, Trebuchon-Da Fonseca A, et al. Hippocampal Interictal Spikes during Sleep Impact Long-Term Memory Consolidation. Ann Neurol. 2020;87(6):976-87.

27. Risher WC, Eroglu C. Thrombospondins as key regulators of synaptogenesis in the central nervous system. Matrix Biol. 2012;31(3):170-7.

28. Jayakumar AR, Apeksha A, Norenberg MD. Role of Matricellular Proteins in Disorders of the Central Nervous System. Neurochem Res. 2017;42(3):858-75.

29. Wang F, Shen X, Li S, Chen L, Wang Y, Qin J, et al. Splenocytes derived from young WT mice prevent AD progression in APPswe/PSENIdE9 transgenic mice. Oncotarget. 2015;6(25):20851-62.

30. Schafer MJ, LeBrasseur NK. The influence of GDF11 on brain fate and function. Geroscience. 2019;41(1):1-11.

31. Yang R, Fu S, Zhao L, Zhen B, Ye L, Niu X, et al. Quantitation of circulating GDF-11 and beta2-MG in aged patients with age-related impairment in cognitive function. Clin Sci (Lond). 2017;131(15):1895904.

32. Choi C, Jeong JH, Jang JS, Choi K, Lee J, Kwon J, et al. Multiplex analysis of cytokines in the serum and cerebrospinal fluid of patients with Alzheimer's disease by color-coded bead technology. J Clin Neurol. 2008;4(2):84-8.

33. Butcher L, Peres K, Andre P, Morris RH, Walter S, Dartigues JF, et al. Association between plasma CCL11 (eotaxin-1) and cognitive status in older adults: Differences between rural and urban dwellers. Exp Gerontol. 2018;113:173-9.

34. Erickson MA, Morofuji Y, Owen JB, Banks WA. Rapid transport of CCL11 across the blood-brain barrier: regional variation and importance of blood cells. J Pharmacol Exp Ther. 2014;349(3):497507.

\section{Figures}



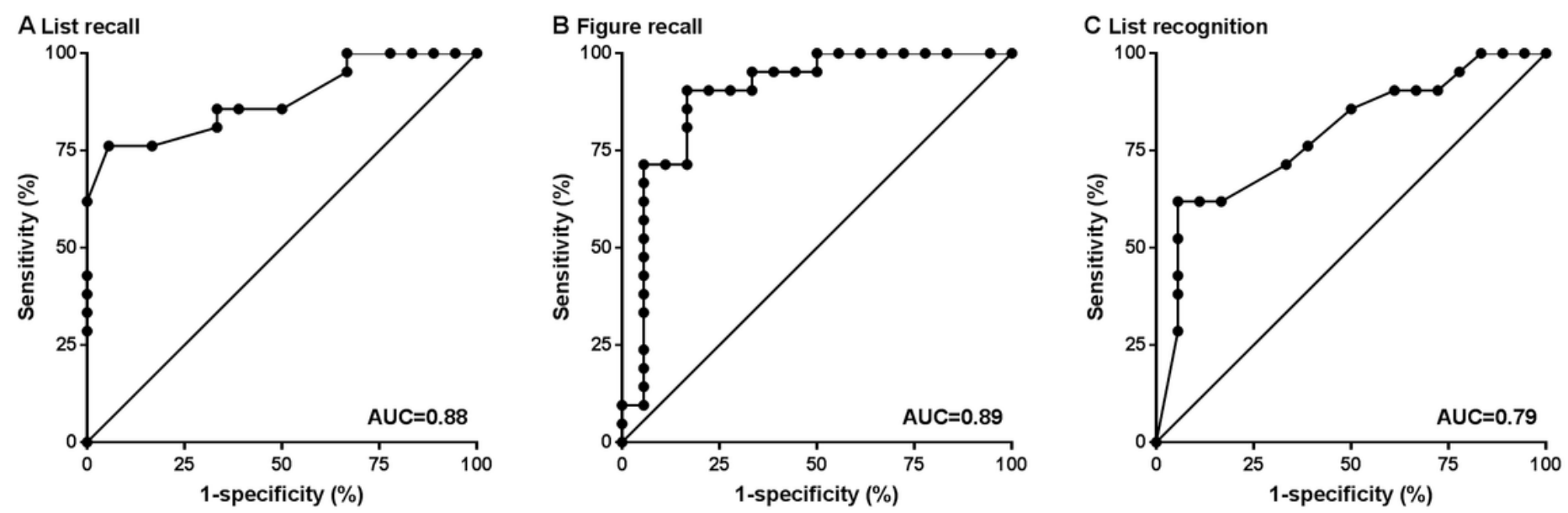

Figure 1

ROC curves for long-term forgetting rates as a discriminator between preclinical mutation carriers and non-carriers ROC curves are shown for long-term forgetting rates for (A) list recall, (B) figure recall and (C) list recognition.
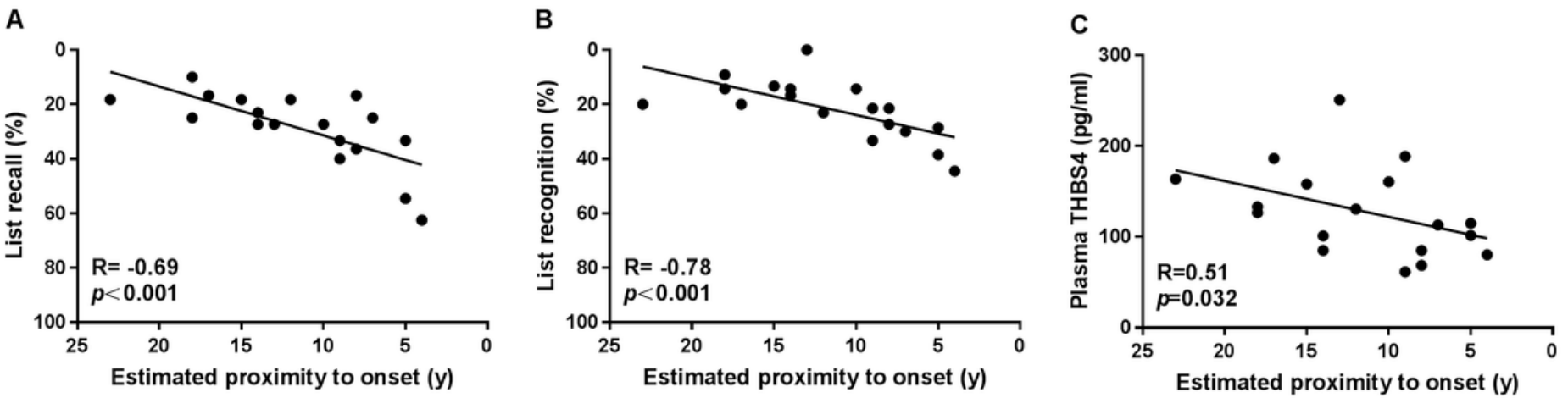

Figure 2

Scatter plots for estimated proximity to onset against long-term forgetting rates and blood-borne factors in mutation carriers. Association between estimated proximity to onset and (A) forgetting rate in list recall, (B) forgetting rate in list recognition, and (C) plasma THBS4 concentration.

\section{Supplementary Files}

This is a list of supplementary files associated with this preprint. Click to download.

- SupplementaryData.docx 